\title{
High-tech adjuncts to emergency weight estimation: Point-of-care ultrasound and point-of-care bioelectrical impedance measurements can increase the accuracy of length-based weight estimation in children
}

\author{
Mike Wells ${ }^{1 *}$, Lara Goldstein ${ }^{1}$, and Alison Bentley ${ }^{1}$ \\ ${ }^{1}$ Division of Emergency Medicine, Faculty of Health Sciences, University of the Witwatersrand, South Africa
}

\begin{abstract}
Introduction: An accurate and reliable method of estimating weight in sick obese children, for purposes of drug dosing calculations, has proved to be elusive. Incorporating the use of high-tech, point-of-care technology into weight-estimation methodology has not been evaluated before. We aimed to determine if ultrasound and bioelectrical impedance measurements could be used in combination with the PAWPER XL tape (PTXL) to increase the accuracy of total body weight (TBW), ideal body weight (IBW) and fat-free mass (FFM) estimation.

Methods: A convenience sample of 332 children attending an academic Emergency Department was enrolled. Each child had their weight estimated with the PAWPER XL tape and underwent anthropometric, point-of-care ultrasound (PoCUS) and bioelectrical impedance (BI) measurements of parameters useful for body composition assessment. Dual X-ray absorptiometry (DXA) was measured and used as a reference for body composition. Multiple regression techniques were used to identify variables and variable-combinations that were strongly associated with TBW, IBW and DXA-measured FFM. Low-tech combinations including anthropometric measurements only were compared with models including high-tech measurements.
\end{abstract}

Results: The PTXL alone, with no low- or high-tech adjunct, provided the most accurate estimate of IBW. Estimates of TBW based on anthropometric measurements were significantly improved with the addition of poCUS or BI to the prediction models, especially in underweight and obese children. Similarly, high-tech models significantly outperformed the low-tech models for estimating FFM.

Conclusions: The use of PoCUS and BI significantly improved the accuracy of TBW and FFM estimation in children when compared with anthropometric-based methods.

\section{Introduction}

Why is an accurate estimation of weight crucially important during the management of medical emergencies in children? During emergencies, actual weight generally cannot be measured and, if medications are required, then an estimate of weight is required to enable the calculation of drug doses [1]. It follows that if the weight estimate is not accurate then the resulting drug dose will not be accurate, which may result in patient harm [2-4]. The use of weight-estimation techniques known to be inaccurate should therefore not be tolerated the most accurate systems that are practical during emergencies should be utilised $[5,6]$.

The newer, dual length- and habitus-based weight estimation systems, such as the PAWPER XL tape (PTXL), have been shown to be significantly more accurate than older methods in underweight and obese children, primarily because they account for variations in body habitus [7-9]. If systems that estimate habitus are accurate, then perhaps techniques that directly measure body composition could be even more accurate.

There is little data on the role of technology in paediatric weight estimation, but there are several significant ways in which high-tech solutions could assist weight estimation and emergency drug dose calculations in children.
- $\quad$ Specialised in-stretcher scales could be used to measure total body weight (TBW) in an emergency. The accuracy of this type of equipment during emergencies has, surprisingly, not been validated. The limited available evidence suggests that medical management frequently prevents these scales from being used [10]. Furthermore, scales can only provide a measure of TBW; if a measure of ideal body weight (IBW) was required for obese children then this would still have to be estimated.

- $\quad$ Computerised point-of-care systems or smartphone apps are a valuable adjunct to emergency medical management. These systems can eliminate the need for calculations for weight-estimation systems in addition to providing comprehensive drug-dosing information [11]. The use of such devices could also allow for more complex weight estimation methodologies to be used without generating a debilitating cognitive burden.

Correspondence to: Mike Wells, Division of Emergency Medicine, Faculty of Health Sciences, University of the Witwatersrand, 5 York Road, Parktown Johannesburg, South Africa, Tel: +27824910369; E-mail: mike.wells@emergencymedicine.co.za

Key words: emergency weight estimation, medical emergencies, emergency department, body weight, fat-free mass

Received: March 18, 2017; Accepted: April 17, 2017; Published: April 20, 2017 
Wells M (2017) High-tech adjuncts to emergency weight estimation: Point-of-care ultrasound and point-of-care bioelectrical impedance measurements can increase the accuracy of length-based weight estimation in children

- High-tech systems could, at least in theory, also be used to rapidly generate additional data that might supplement or replace anthropometric measurements to increase the accuracy of weight estimation - especially in children at risk for critical weight estimation errors (obese and underweight children). Point-of-care ultrasound (PoCUS) and bioelectrical impedance (BI) have been validated for body composition assessment in children $[12,13]$. These techniques could potentially be adapted to provide an indication of a child's body composition to allow for an accurate, individualised estimation of total body TBW, IBW or even fat-free mass (FFM). Either IBW or FFM is required for correct dosing of hydrophilic medications in obese children [14].

The aim of this study was to evaluate whether commonly available, high-tech point-of-care resources, such as PoCUS and BI, would be able to improve the accuracy of the PAWPER XL tape with respect to TBW, IBW and FFM estimations in obese, underweight and normal weight children, when compared to low-tech anthropometrical methods.

\section{Methods}

\section{Study design and setting}

This was a prospective, cross-sectional study conducted in the Emergency Department (ED) of an academically-aligned private hospital in Johannesburg, South Africa. Ethics approval was obtained from the Human Research Ethics Committee of the University of the Witwatersrand.

\section{Selection of participants}

A convenience sample of 332 children aged from one month to 16 years who presented to the ED between October and December 2014 were enrolled. Only children whose participation did not interfere with medical treatment were eligible for inclusion.

\section{Methods and measurements}

Data was collected by one of the researchers (MW or LG) using the following procedure for each child:

- Basic demographic data was obtained and recorded.

- The child was positioned supine on the examination bed (to simulate positioning during emergency care) for weight estimation by the PAWPER XL tape. The PTXL uses length and a visual assessment of habitus to generate an estimate of TBW and length alone to generate an estimate of IBW.

- Basic anthropometric measurements: length, mid-arm circumference (MAC), humerus length, ulna length, tibia length, waist circumference, hip circumference and thigh circumference were also obtained.

- B-mode ultrasound measurements of the subcutaneous fatlayer thickness and muscle thickness were taken from the anterior aspects of the mid-arm (biceps) and mid-thigh (quadriceps) using a Mindray DP50 with a $7 \mathrm{MHz}$ linear probe. Transverse diameters of the midshaft humerus and femur were also measured (see Figure 1). Repeat measurements were made by a second researcher in 31 children to determine inter-observer reliability.

- The child was then weighed on a calibrated digital scale with a foot-to-foot bioelectrical impedance measurement obtained simultaneously (Tanita SC-240 Body Composition Analyser).

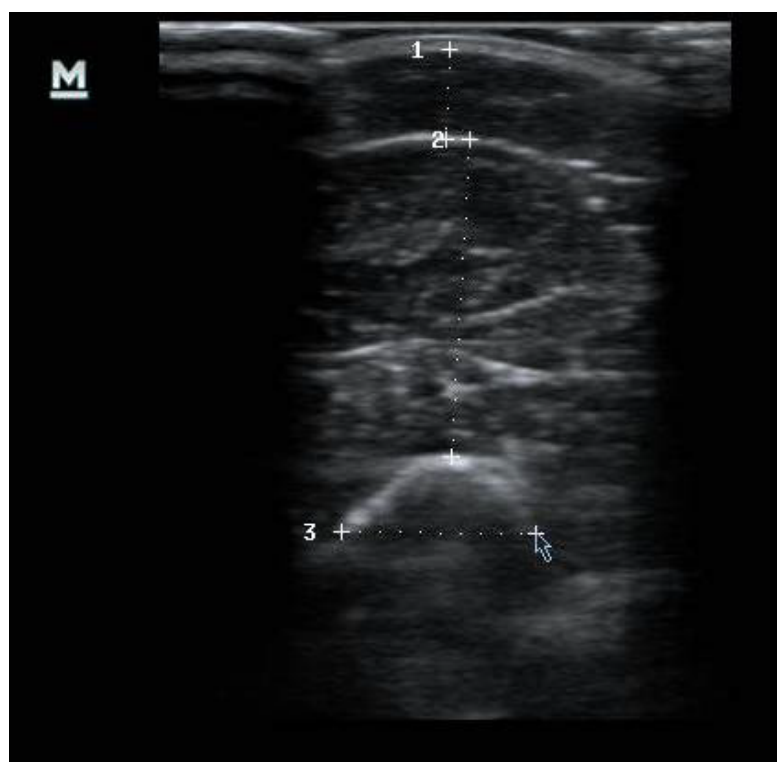

Figure 1. B-mode ultrasound image of biceps. This is a transverse view of the midarm, with zero pressure applied to the skin. The measurement marked "1" represents the subcutaneous fat layer thickness, the measurement marked " 2 " demonstrates the muscle layer thickness and " 3 " the transverse diameter of the mid-humerus.

- Finally, whole-body dual X-ray absorptiometry (DXA) measurements of body composition were obtained using a Hologic Discovery A Densitometer with paediatric software, version 12.6.

The demographic data, anthropometric measurements, BI data and DXA data were used to calculate several additional and derivative variables and used in previously described body compositionpredicting formulas (see Table 1). IBW was calculated for each child using the BMI method [15].

\section{Analysis}

Inter-observer reliability for PoCUS was evaluated using intra-class correlation (ICC).

The relationships between TBW, IBW and DXA-measured fatfree mass (FFM) as individual dependent variables and multiple anthropometric, PoCUS and BI measurements were evaluated using linear and non-linear regression techniques. Those variables with the highest predictive outcomes, as determined by the $\mathrm{R}^{2}$ and root mean squared error (RMSE) results were evaluated in combination with the PTXL data and anthropometric data to obtain the best possible multivariate predictive models of TBW, IBW and FFM.

\section{Model development rules, assumptions and outcome measures}

We assumed that a point-of-care app would be available to perform all complex calculations - this allowed for more complex models, while keeping the input simple.

These best-performing models were used to generate estimations of TBW, IBW for all children and FFM for obese children only. From this data, the proportions of estimates falling within $10 \%$ (PW10) of the target weight were calculated and then compared using the Fisher exact test to establish significant differences in accuracy. A PW 10>70\% was considered a benchmark for satisfactory model performance.

\section{Software}

Statistical analysis was performed using Microsoft excel (Microsoft. 
Wells M (2017) High-tech adjuncts to emergency weight estimation: Point-of-care ultrasound and point-of-care bioelectrical impedance measurements can increase the accuracy of length-based weight estimation in children

Table 1. Measurements and derived variables evaluated by regression analysis. All anthropometric measurements were performed according to the International Standards for Anthropometric Assessment 2006 guidelines. Indexes of selected variable were calculated as quotients based on height-squared $\left(\mathrm{m}^{2}\right)$. Abbreviations: TBW $=$ total body weight; IBW $=$ ideal body weight; $\mathrm{BMI}=$ body mass index; MAC $=$ mid-arm circumference; $\mathrm{SC}=$ subcutaneous fat layer; $\mathrm{MM}=$ muscle layer; $\mathrm{Z}=$ resistance; $\mathrm{ZI}(\mathrm{Resistance} \mathrm{Index})=$ height $(\mathrm{cm})^{2} / \mathrm{resistance}(\mathrm{Ohms}) ; \mathrm{W}=$ weight in $\mathrm{kg} ; \mathrm{H}=$ height in $\mathrm{cm} ; \mathrm{A}=$ age in years; $\mathrm{S}=\operatorname{sex}($ males $=1$, females $=0)$

\begin{tabular}{|c|c|c|}
\hline PAWPER XL tape measurements & \multicolumn{2}{|c|}{ TBW (from length and habitus) and IBW (from length) estimations } \\
\hline Anthropometric measurements & \multicolumn{2}{|c|}{$\begin{array}{l}\text { Recumbent length, humerus length, ulna length, tibia length, mid-arm circumference, waist circumference, hip circumference, thigh } \\
\text { circumference }\end{array}$} \\
\hline Derived anthropometric variables & \multicolumn{2}{|c|}{ BMI, BMI-for-age Z-score, BMI-IBW, MAC-index, waist-index, hip-index, waist: hip ratio } \\
\hline Ultrasound measurements & \multicolumn{2}{|c|}{$\begin{array}{l}\text { Mid-arm subcutaneous fat layer (SC), biceps thickness (MM), mid-humerus transverse diameter, mid-thigh (quadriceps) SC, quadriceps MM, } \\
\text { mid-femur transverse diameter }\end{array}$} \\
\hline Derived ultrasound variables & \multicolumn{2}{|c|}{$\begin{array}{l}\text { Biceps SC index, biceps MM index, humerus diameter index, biceps SC:MM ratio, quadriceps SC index, quadriceps MM index, femur } \\
\text { diameter index, quadriceps SC:MM ratio, sum of biceps and quadriceps SC thickness }\end{array}$} \\
\hline Bioelectrical impedance measurements & \multicolumn{2}{|l|}{ Foot-to-foot impedance (resistance) at $50 \mathrm{~Hz}$} \\
\hline $\begin{array}{l}\text { Fat-free mass estimations from } \\
\text { bioelectrical impedance measurements } \\
\text { and anthropometric measurements }\end{array}$ & $\begin{array}{l}\text { Clasey formula: } \\
\text { Schaefer formula: } \\
\text { Houtkooper formula: } \\
\text { Deurenberg formula: } \\
\text { Cordain formula: } \\
\text { Tyrell formula: } \\
\text { Rush formula: } \\
\text { Dung formula: } \\
\text { De Lorenzo formula: } \\
\text { Davies formula 1: } \\
\text { Fjeld formula: } \\
\text { Gregory formula: } \\
\text { Davies formula 2: } \\
\text { Danford formula: } \\
\text { Kushner formula 1: } \\
\text { Kushner formula 2: Foster formula for } \\
\text { FFM for boys } \\
\text { Foster formula for FFM for girls }\end{array}$ & $\begin{array}{l}\mathrm{FFM}=(-7.655+297 \mathrm{H}+125 \mathrm{~W}-17.4 \mathrm{Z}) / 1000 \\
\mathrm{FFM}=0.15+0.65 \mathrm{Z}+0.68 \mathrm{~A} \\
\mathrm{FFM}=0.61 \mathrm{ZI}+0.25 \mathrm{~W}+1.31 \\
\mathrm{FFM}=0.406 \mathrm{Z}+0.36 \mathrm{~W}+0.0558 \mathrm{H}+0.56 \mathrm{~S}-6.48 \\
\mathrm{FFM}=6.86+0.81 \mathrm{ZI} \\
\mathrm{FFM}=0.31 \mathrm{ZI}+0.17 \mathrm{H}+0.11 \mathrm{~W}+0.942 \mathrm{~S}-14.96 \\
\mathrm{FFM}=0.622 \mathrm{ZI}+0.234 \mathrm{~W}+1.166 \\
\mathrm{FFM}=0.786 \mathrm{Z}+0.093 \mathrm{~W}+0.194 \\
\mathrm{FFM}=2.330+0.588 \mathrm{ZI}+0.211 \mathrm{~W} \\
\text { Total body water }=-0.50+0.60 \mathrm{ZI} \\
\text { Total body water }=0.76+0.18 \mathrm{ZI}+0.39 \mathrm{~W} \\
\text { Total body water }=0.79+0.55 \mathrm{ZI} \\
\text { Total body water }=0.13+0.58 \mathrm{ZI} \\
\text { Total body water }=1.84+0.45 \mathrm{ZI}+0.11 \mathrm{~W} \\
\text { Total body water }=0.700 \mathrm{ZI}-0.32 \\
\text { Total body water }=0.593 \mathrm{ZI}+0.065 \mathrm{~W}+0.04 \\
\text { FFM }=\exp \left(-2.899+0.8064 \times \operatorname{lnH}+0.5674 \times \operatorname{lnW}+0.0000185 \times \mathrm{W}^{2}-0.0153 \times \mathrm{Z} \text {-score }{ }^{2}+0.0132 \times \mathrm{A}\right. \\
\text { FFM }=\exp \left(-3.8345+0.954 \times \ln \mathrm{H}+0.6515 \times \ln \mathrm{W}-0.0102 \times \text { Zcore }^{2}\right.\end{array}$ \\
\hline
\end{tabular}

Notes:

Measured weight was replaced with PTXL-total body weight

Measured height was replaced with height obtained from the PTXL length

Age was replaced with age at the $50^{\text {th }}$ centile of length-for-age obtained from the PTXL length

Total body water was converted to FFM by dividing by $0.76(0.76 \mathrm{~mL} / \mathrm{g})$ in children from 5 to 16 years, and 0.78 for children below 5 years of age.

Microsoft Excel. Redmond, Washington: Microsoft, 2016) and Stata (StataCorp. 2015. Stata Statistical Software: Release 14. College Station, TX: StataCorp LP).

\section{Results}

\section{Study population}

A total of 332 children were enrolled in the study. The basic demographic information is shown in Table 2. The sample showed a reasonable number of underweight children (15.3\%) and overweight or obese children (32.5\%) in which to evaluate the anthropometric and high-tech weight-estimation models. The breakdown by weightstatus showed reasonably well-matched groups, except for a high proportion of very young children in the severely obese category. The anthropometric, PoCUS and BI data all showed a strong association with body habitus.

\section{PoCUS, BI and body composition}

The relationships between PoCUS measurements of subcutaneous fat and muscle layers and body fat (BF) and FFM are shown in Figure 2. The biceps measurements had a higher predictive power than those from the quadriceps site. The inter-observer reliability of the PoCUS measurements was exceptionally good, with ICCs ranging from 0.984 to 0.991 for the different sites.

The scatterplots of BI against body fat and FFM can also be found in Figure 2. The best relationship was found between a logarithmically transformed resistance index (since the relationship between length and weight is not linear) and FFM.

\section{Single variable regression outcomes}

The results of single-variable linear regression with TBW, IBW and FFM as dependant variables are shown in Table 3. There were strong associations between humerus length, MAC, hip circumference, recumbent length and impedance index and all dependant variables. There was a very strong association between PTXL-predicted IBW and calculated IBW. Ultrasound measurements, as single variables, were only weakly associated with any of the dependant variables. The BI FFM formulas, with data imputed from PTXL estimations, were strongly predictive of FFM.

\section{Combination variable regression outcomes}

Only outcomes that achieved an adjusted- $\mathrm{R}^{2}$ of more than 0.95 and a RMSE of less than $3.2 \mathrm{~kg}$ were included in the results (see Table 4). Both PoCUS and BI demonstrated an ability to improve the predictive ability of length-based weight estimation. There were several high-tech combinations that showed good predictive value for TBW and FFM. The PTXL, as a single variable, predicted IBW very accurately and no additional variables increased the accuracy of this prediction.

\section{Comparisons between high-tech and low-tech estimation outcomes}

Each one of the high-tech models of TBW-estimation was significantly more accurate than the purely anthropometric combinations, with odds ratios ranging from 1.6 to 2.9. This amounted to an $8-16 \%$ absolute improvement in weight estimation PW10 accuracy. Both the low-tech and the high-tech models predicted FFM accurately, but the high-tech models were significantly better than the low-tech models, although with a small effect size. 
Wells M (2017) High-tech adjuncts to emergency weight estimation: Point-of-care ultrasound and point-of-care bioelectrical impedance measurements can increase the accuracy of length-based weight estimation in children
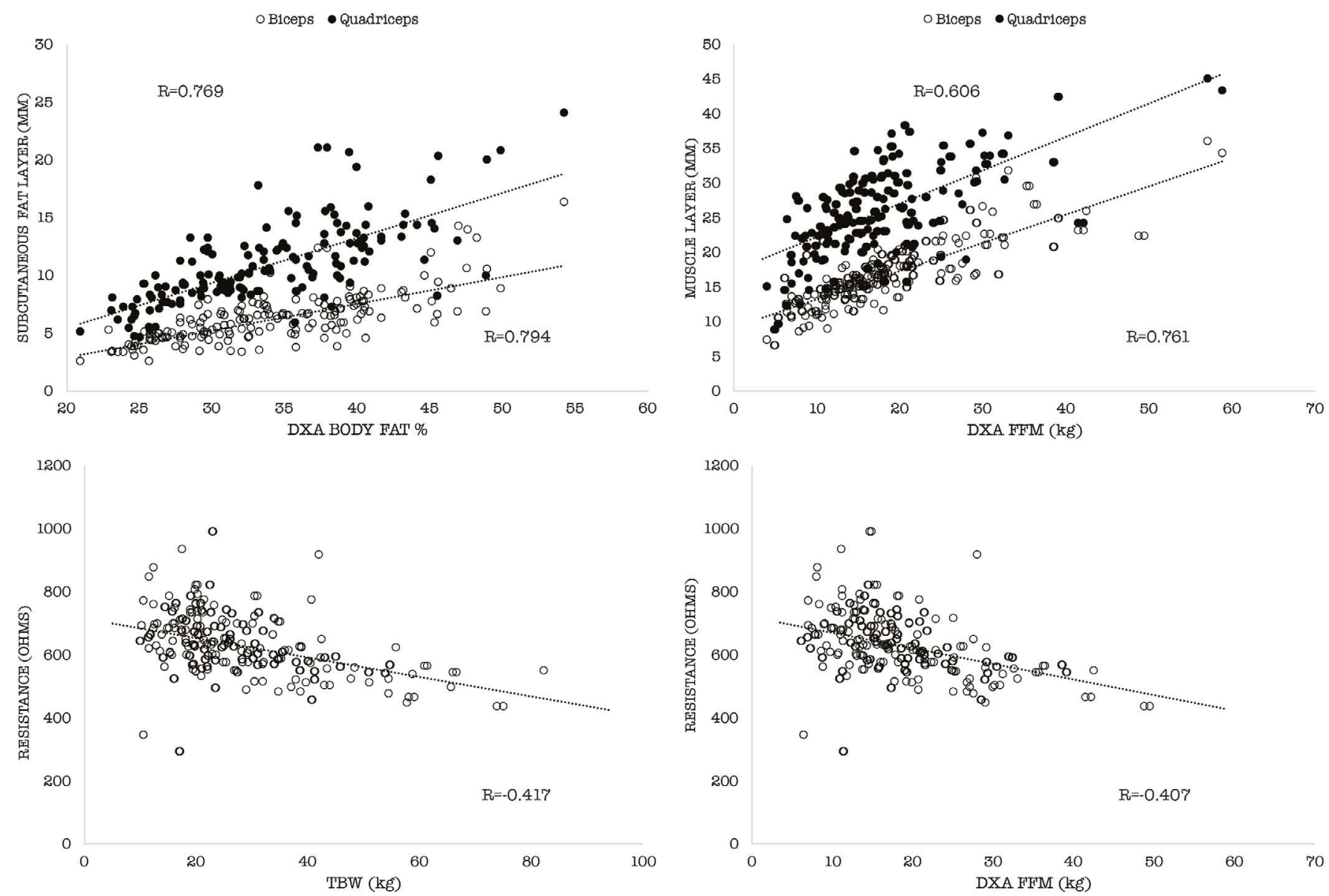

Figure 2. Scatterplots of PoCUS and bioelectrical impedance measurements against body composition variables. The correlation coefficients for each pairing are shown (Pearson's correlation, $\mathrm{p}<0.001$ for each).

Table 2. Demographic data for the study population. The data is shown for all patients and for subgroups of habitus (stratified by BMI-for-age Z scores). The PTXL habitus score is on a scale of 1 to 7 with HS1 representing an underweight child, HS3 a $50^{\text {th }}$ centile child and HS7 a severely obese child. All data is presented with medians and interquartile range unless otherwise specified. Abbreviations: $\mathrm{BMI}=$ body mass index, $\mathrm{BF}=$ body fat; FMI $=$ fat mass index, FFMI $=$ fat-free mass index, $\mathrm{IBW}=\mathrm{ideal}$ body weight, $\mathrm{PTXL}=\mathrm{PAWPER}$ XL tape, $\mathrm{TBW}=$ total body weight, $\mathrm{MAC}=$ mid-arm circumference, $\mathrm{US}=$ ultrasound, $\mathrm{SC}=$ subcutaneous fat layer, $\mathrm{MM}=$ muscle layer.

\begin{tabular}{|c|c|c|c|c|c|c|c|}
\hline & All & $\begin{array}{c}\text { Underweight } \\
Z \leq-2.0\end{array}$ & $\begin{array}{c}\text { Thin } \\
-2.0<\mathrm{Z} \leq-1.4\end{array}$ & $\begin{array}{c}\text { Normal } \\
-1.4<Z<-1.4\end{array}$ & $\begin{array}{l}\text { Overweight } \\
1.4 \leq Z<2.0\end{array}$ & $\begin{array}{c}\text { Obese } \\
2.0 \leq Z<2.5\end{array}$ & $\begin{array}{c}\text { Severely obese } \\
\quad Z \geq 2.5\end{array}$ \\
\hline Number n (\%) & 332 & $18(5.4)$ & $33(9.9)$ & $173(52.1)$ & $74(22.3)$ & $20(6.0)$ & $14(4.2)$ \\
\hline Sex (male) n (\%) & $154(46.4)$ & $4(22.2)$ & $15(45.5)$ & $93(53.7)$ & $25(33.8)$ & $12(60.0)$ & $5(35.7)$ \\
\hline Age (years) & $7.2(4.5,9.3)$ & $6.4(3.5,8.0)$ & $9.1(5.7,13.1)$ & $7.3(4.9,10.1)$ & $7.8(5.0,9.4)$ & $6.7(4.9,10.0)$ & $3.0(1.7,6.9)$ \\
\hline BMI $\left(\mathrm{kg} / \mathrm{m}^{2}\right)$ & $16.7(15.2,18.8)$ & $13.2(12.9,13.4)$ & $16.3(15.6,18.6)$ & $16.2(15.4,17.3)$ & $18.6(17.8,20.0)$ & $19.9(19.3,26.6)$ & $22.6(20.5,26.4)$ \\
\hline BMI-for-age Z-score & $0.4(-0.5,1.1)$ & $-2.7(-3.5,-2.2)$ & $-1.7(-1.8,-1.5)$ & $0.3(-0.3,0.6)$ & $1.4(1.1,1.6)$ & $2.1(2.0,2.2)$ & $2.9(2.8,3.5)$ \\
\hline BF (\%) & $27.7(24.2,32.0)$ & $23.2(21.4,26.7)$ & $26.8(24.6,27.7)$ & $25.5(23.4,28.6)$ & $32.3(29.7,33.7)$ & $34.8(31.3,37.8)$ & $38.3(34.9,45.2)$ \\
\hline FMI $\left(\mathrm{kg} / \mathrm{m}^{2}\right)$ & $4.6(3.7,5.7)$ & $3.0(2.7,3.4)$ & $4.5(4.3,4.7)$ & $4.2(3.7,4.9)$ & $5.9(5.5,6.7)$ & $6.8(6.1,10.3)$ & $8.8(7.1,11.2)$ \\
\hline FFMI $\left(\mathbf{k g} / \mathbf{m}^{2}\right)$ & $12.2(11.3,13.2)$ & $10.0(9.6,10.3)$ & $11.8(11.5,14.1)$ & $12.1(11.5,12.7)$ & $12.8(12.3,14.1)$ & $14.4(12.6,15.1)$ & $13.9(13.4,15.2)$ \\
\hline IBW (kg) & $23.2(17.7,30.7)$ & $22.2(14.9,23.5)$ & $26.4(21.2,43.0)$ & $22.8(18.3,31.5)$ & $25.9(18.9,31.5)$ & $23.7(18.2,35.9)$ & $14.2(11.3,35.9)$ \\
\hline PTXL Habitus Score & $3(3,4)$ & $2(2,2)$ & $3(3,3.8)$ & $3(3,4)$ & $4(4,4.8)$ & $4.5(4,5)$ & $5(5,6)$ \\
\hline TBW (kg) & $23.4(17.6,33.8)$ & $18.3(12.3,20.0)$ & $26.6(21.1,43.1)$ & $23.4(18.4,31.4)$ & $30.6(21.6,38.8)$ & $30.8(21.3,56.3)$ & $19.0(15.5,39.3)$ \\
\hline MAC (cm) & $19.0(17.0,22.0)$ & $16.0(14.8,17.5)$ & $17.3(16.0,18.5)$ & $19.0(17.5,21.5)$ & $22.5(21.3,26.8)$ & $22.3(20.4,30.3)$ & $21.8(19.1,26.1)$ \\
\hline Resistance ( $\Omega$ ) & $626(568,690)$ & $762(683,806)$ & $727(686,771)$ & $627(571,681)$ & $567(539,591)$ & $566(497,607)$ & $516(483,558)$ \\
\hline $\begin{array}{l}\text { Resistance index } \\
\left(\mathrm{cm}^{2} / \Omega\right)\end{array}$ & $23.2(17.8,31.5)$ & $17.3(14.8,19.6)$ & $19.7(17.7,24.7)$ & $23.3(17.7,31.6)$ & $28.5(23.8,39.9)$ & $24.9(20.7,38.5)$ & $18.2(12.8,33.1)$ \\
\hline US biceps SC (mm) & $6.0(4.7,7.2)$ & $4.4(3.5,4.9)$ & $4.7(4.3,4.8)$ & $5.5(4.4,6.6)$ & $7.3(6.6,8.7)$ & $7.4(7.0,10.1)$ & $8.2(7.5,10.5)$ \\
\hline US biceps MM (mm) & $16.1(13.7,18.6)$ & $12.6(7.4,15.7)$ & $13.8(11.3,15.2)$ & $15.5(13.1,17,6)$ & $19.6(17.3,22.4)$ & $19.5(18.0,21.7)$ & $14.1(13.8,21.2)$ \\
\hline $\begin{array}{c}\text { US quadriceps SC } \\
(\mathbf{m m})\end{array}$ & $10.1(8.6,12.6)$ & $8.3(6.1,10.0)$ & $5.5(4.8,8.4)$ & $9.5(8.0,11.7)$ & $13.0(11.7,14.9)$ & $10.0(8.0,13.7)$ & $17.1(15.2,20.5)$ \\
\hline $\begin{array}{c}\text { US quadriceps MM } \\
(\mathrm{mm})\end{array}$ & $25.5(22.2,30)$ & $21.8(12.3,22.9)$ & $23.9(19.7,27.7)$ & $24.5(20.1,29.1)$ & $28.8(23.6,34.1)$ & $19.2(17.4,27.6)$ & $26.4(17.8,27.8)$ \\
\hline
\end{tabular}


Wells M (2017) High-tech adjuncts to emergency weight estimation: Point-of-care ultrasound and point-of-care bioelectrical impedance measurements can increase the accuracy of length-based weight estimation in children

Table 3. Outcome data of linear regression for individual variables and impedance formulas. An $\mathrm{R}^{2}>0.95$ and a RMSE of $<5 \mathrm{~kg}$ was considered to represent a useful predictive ability Abbreviations: TBW $=$ total body weight, IBW $=$ ideal body weight, $\mathrm{FFM}=$ fat-free mass, $\mathrm{RMSE}=$ root mean squared error, $\mathrm{MAC}=$ mid-arm circumference, $\mathrm{PTXL}=\mathrm{PAWPER}$ XL tape, $\mathrm{SC}=$ subcutaneous fat layer, $\mathrm{MM}=$ muscle layer.

\begin{tabular}{|c|c|c|c|c|c|c|c|c|c|}
\hline \multirow[b]{2}{*}{ Variable } & \multicolumn{3}{|c|}{ TBW } & \multicolumn{3}{|c|}{ IBW } & \multicolumn{3}{|c|}{ FFM } \\
\hline & $\mathbf{R}^{2}$ & RMSE (kg) & $\mathbf{F}$ & $\mathbf{R}^{2}$ & RMSE (kg) & $\mathbf{F}$ & $\mathbf{R}^{2}$ & RMSE (kg) & $\mathbf{F}$ \\
\hline Age & 0.74 & 7.3 & 923 & 0.91 & 3.3 & 3260 & 0.83 & 3.9 & 1594 \\
\hline Humerus & 0.77 & 6.8 & 1123 & 0.88 & 3.8 & 2383 & 0.84 & 3.8 & 1696 \\
\hline Tibia & 0.67 & 8.2 & 660 & 0.84 & 4.3 & 1785 & 0.76 & 4.6 & 1043 \\
\hline Ulna & 0.72 & 7.5 & 843 & 0.83 & 4.5 & 1602 & 0.79 & 4.3 & 1215 \\
\hline MAC & 0.89 & 4.7 & 2652 & 0.64 & 6.5 & 585 & 0.78 & 4.4 & 1164 \\
\hline Waist circumference & 0.74 & 7.2 & 931 & 0.48 & 7.8 & 305 & 0.62 & 5.7 & 527 \\
\hline Hip circumference & 0.89 & 4.6 & 2725 & 0.72 & 5.7 & 844 & 0.81 & 4.0 & 1411 \\
\hline Thigh circumference & 0.78 & 6.6 & 1172 & 0.61 & 6.8 & 507 & 0.70 & 5.1 & 762 \\
\hline Length & 0.75 & 7.2 & 964 & 0.87 & 3.9 & 2281 & 0.82 & 3.9 & 1521 \\
\hline Habitus Score & 0.19 & 12.8 & 77 & 0.02 & 10.8 & 7 & 0.09 & 8.9 & 32 \\
\hline PTXL-IBW & 0.87 & 5.1 & 2245 & 0.98 & 1.6 & 15599 & 0.95 & 2.1 & 6474 \\
\hline PTXL-TBW & 0.97 & 2.5 & 9937 & 0.88 & 3.8 & 2460 & 0.95 & 2.2 & 5815 \\
\hline US biceps SC & 0.14 & 13.2 & 49 & NS & & & 0.05 & 9.2 & 15 \\
\hline US biceps MM & 0.62 & 8.8 & 489 & 0.49 & 7.9 & 292 & 0.58 & 6.1 & 412 \\
\hline US thigh SC & 0.16 & 12.2 & 54 & 0.01 & 10.6 & 8 & 0.07 & 8.8 & 22 \\
\hline US thigh MM & 0.35 & 10.7 & 158 & 0.31 & 8.9 & 133 & 0.37 & 7.2 & 166 \\
\hline Resistance & 0.17 & 12.8 & 67 & 0.08 & 10.4 & 27 & 0.17 & 8.5 & 63 \\
\hline Resistance index & 0.85 & 5.4 & 1819 & 0.83 & 4.4 & 1593 & 0.90 & 2.9 & 2940 \\
\hline Houtkooper formula & & & & & & & 0.95 & 2.1 & 6022 \\
\hline Rush formula & & & & & & & 0.95 & 2.1 & 5787 \\
\hline deLorenzo formula & & & & & & & 0.95 & 2.1 & 5664 \\
\hline Fjeld formula & & & & & & & 0.97 & 1.7 & 9120 \\
\hline Foster formula & & & & & & & 0.96 & 1.8 & 8982 \\
\hline
\end{tabular}

Variables not found to have useful predictive value: all anthropometric and ultrasound measurements normalised for height (e.g. MAC-index MAC/ht ${ }^{2}$ ), ultrasound measurements of humerus and femur diameters; ratios between SC and MM layers and between SC and bone diameters; sum of SC or MM thicknesses.

Formulas not found to have useful predictive value: Clasey formula, Schaefer formula, Deurenberg formula 2, Cordain formula, Tyrell formula, Dung formula, Davies formulas, Gregory formula, Danford formula, Kushner formulas.

\section{Discussion}

This study is the first to have evaluated the theoretical ability of high-tech, point-of-care methodologies as adjuncts to improve the accuracy of TBW, IBW and FFM estimations in children.

\section{Main results}

The high degree of weight-estimation accuracy achieved in this study, using high-tech-enhanced methods, was interesting and warrants further future investigation. The anthropometric measurements generally showed a higher predicative capability than ultrasound or BI when evaluated as a single variable, but in combination with the PTXL and habitus score or MAC, however, PoCUS and BI measurements could significantly increase the accuracy of estimates of both TBW and FFM especially in underweight and obese children.

\section{TBW estimation}

It was in the accuracy of estimation of TBW that the high-tech methods produced the best and most unexpected results. In this study, the accuracy of TBW estimation with the derived regression equations using anthropometric variables was good and was equivalent to the accuracies of the best existing TBW-estimating systems $[7,8,16]$. Both PoCUS and BI, however, when used in combination with the PTXL, significantly and substantially increased the accuracy of TBW estimation, exceeding the accuracy of the low-tech methods [17]. There were strong associations demonstrated between PoCUS measurements of subcutaneous fat and total body fat, PoCUS measurements of muscle thickness and FFM as well as between BI and FFM. This allowed these adjuncts to provide a quantification of FM and FFM when combined with a measurement of length (the PTXL) and thereby increase the accuracy of predictions of TBW.

\section{IBW estimation}

An estimate of IBW is not required for normal-weight or underweight children, as all drug doses in these children should be based on TBW. This is not true for obese children. It is important to safeguard obese children from potentially harmful overdoses with hydrophilic medications such as adrenaline, which would occur if TBW was used as the dose-scalar [14,18]. An estimation of IBW in obese children would therefore be advantageous to allow these drugs to be dosed correctly. The selective use of TBW or IBW as dose-scalars in emergency care has received little attention previously, because existing weight-estimation systems either estimate IBW only (e.g. the Broselow tape) or TBW only (e.g. the Mercy method) [16,19]. Recent advances on the appropriate use of weight-descriptors for hydrophilic and lipophilic drugs in obese children has made a rational dosing strategy now realistically possible [14]. This is important, even in emergencies, as there is potential harm from incorrect dosing $[3,18,20]$.

This study showed that the PTXL predicted IBW exceptionally accurately. This is because IBW is defined by the 50th centile of weightfor-height and the divisions of the PTXL were designed to predict the median weight at any given length $[21,22]$. The addition of other variables could not improve on the accuracy of the PTXL prediction.

\section{FFM estimation}

Whether IBW is an appropriate choice for dose-scaling in obese children has not been fully established and it may be that FFM is a 
Wells M (2017) High-tech adjuncts to emergency weight estimation: Point-of-care ultrasound and point-of-care bioelectrical impedance measurements can increase the accuracy of length-based weight estimation in children

Table 4. Outcome data of linear regression for combinations of low-tech and high-tech variables using the PAWPER XL tape as the method to provide a base of length-based weigh estimation. FFM predictions are only shown for obese children, as this is the only group of children in which an alternative weight descriptor to TBW is required (i.e., a scalar for hydrophilic medications). The superscripts indicate significant outcomes in comparisons between different models. The results of the Fisher exact test, with odds ratios are shown at the bottom of the table. Abbreviations: PTXL = PAWPER XL tape, IBW = ideal body weight, HS = habitus score, MAC $=$ mid-arm circumference, $\mathrm{US}=$ ultrasound, $\mathrm{SC}=$ subcutaneous fat layer, MM = muscle layer, TBW = total body weight, ZI = impedance index, "Rush" = Rush formula for fat-free mass calculation from impedance.

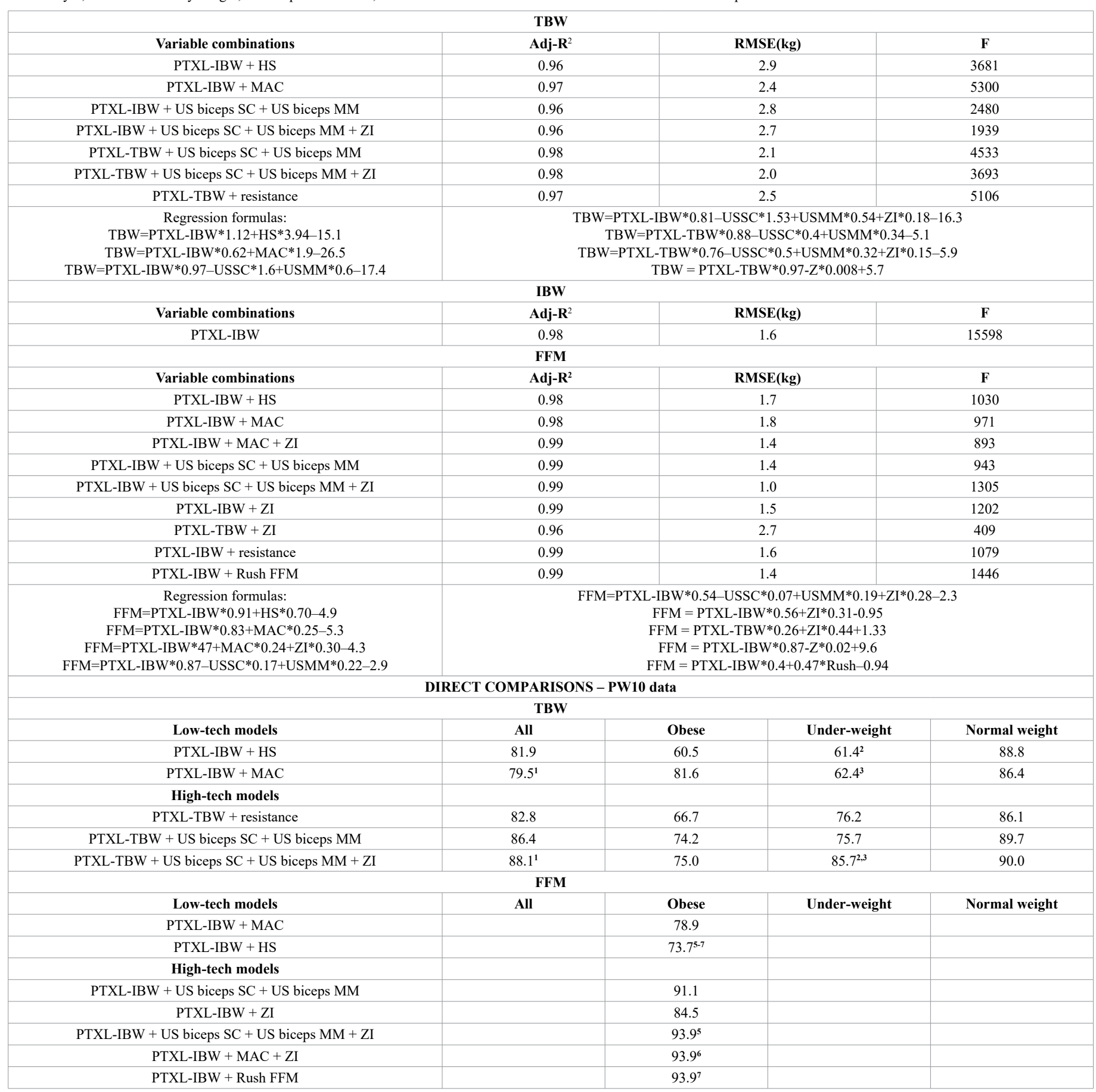

${ }^{1} \mathrm{OR} 1.9(1.2,2.9) \mathrm{p}=0.0044 ;{ }^{2} \mathrm{OR} 4.0(1.4,11.4) \mathrm{p}=0.0142 ;{ }^{3} \mathrm{OR} 3.9(1.3,11.3) \mathrm{p}=0.0131 ;{ }^{5-7} \mathrm{OR} 5.6(1.1,28.2) \mathrm{p}=0.0446$.

more physiologically valid scalar, especially in severely obese children [23]. FFM changes proportionally with the degree of obesity and reflects the volume of distribution of hydrophilic drugs better than IBW [24]. This was the motivation to investigate whether the PTXL could accurately predict FFM in obese children, when combined with low-tech or high-tech adjuncts. While the combination of the PTXL with habitus score or MAC produced accurate estimations of FFM, combining PoCUS measurements and/or BI measurements with the PTXL increased the accuracy of predictions dramatically. These models achieved better results than those previously reported for existing paediatric FFM-estimating formulas (such as the Foster formula) [24,25]. 


\section{PoCUS as an adjunct for assessing body weight}

Ultrasound has been proven to be useful for predicting body composition [12,26,27]. While multiple sites of ultrasound measurement are generally used for body composition analysis, only two easily-accessible sites were used in this study, to simulate real-world access restrictions in sick children. Despite this limitation, and with the use of both subcutaneous fat and muscle thickness measurements, PoCUS showed a strong association with fat and fat-free mass. The relationship between body fat and the subcutaneous fat layer thickness, and between FFM and the muscle layer thickness was stronger at the biceps site than the quadriceps site. This then allowed a single site to provide adequate discriminatory information for the purposes of weight estimation. The ease-of-use and reliability of this technique, the existing evidence on its use in assessing body composition as well as the surprisingly good results in this study make limited B-mode PoCUS a potentially realistic adjunct to weight estimation in the ED or ICU. Although the time taken to obtain the measurements was not recorded, it took only a few seconds to obtain and analyse the images, which would not be an impediment to the potential use of this technique.

\section{Bioelectrical impedance as an adjunct for estimating body weight}

BI also has a very well-established role in the assessment of body composition $[13,20]$. It has never been evaluated with respect to weight estimation, but the relationship between BI and FFM suggests that it might be able to predict overall body size in a way that could improve weight estimations. In this study, the addition of resistance or resistance index to PTXL-based measurement of length allowed for an improvement in TBW and FFM estimation, although primarily when added to PoCUS measurements. BI, when measured using electrodes, could in theory be quickly and easily obtained in the ED or ICU. The usefulness of BI as a high-tech adjunct was overall less than that of PoCUS, however.

\section{Weight estimations in obese, underweight and average habitus children}

The use of the high-tech adjuncts resulted in the greatest improvement in TBW-estimation accuracy in the subgroups of obese and underweight children. These are the children that most often receive inaccurate weight estimations with current weight-estimation systems, which was corroborated by the failure of low-tech methods to achieve the same high level of accuracy as the high-tech methods. This further illustrates the value of a high-tech method that employs a direct measurement of body composition.

\section{Weight estimation during emergencies}

During the stress of paediatric emergencies, the ability of healthcare providers to remember and perform critical actions is seriously impaired [28-30]. Nonetheless, the emergency nature of any presentation must not excuse the delivery of a sub-optimal level of care. The correct level of care demands accurate dosing of all drugs (hydrophilic and lipophilic) in all children (underweight, normal weight and obese children). For this reason, both TBW and IBW (or FFM) are required if medication errors are to be avoided. Since we are compelled to estimate both TBW and IBW accurately, even in emergencies, we may be forced to adopt more advanced techniques, to accommodate technology appropriately and to change our approach to training and practice.

The use of technology, in the form of smartphone apps, to transform complex weight estimation and drug dosing processes into low cognitive-load techniques is of enormous potential benefit. Apps are already an inextricable part of medical practice, and their use may open the door to adopting more sophisticated and complex methods of predicting weight without a negative effect on medical care $[11,31]$. An app which could automatically perform the calculations required for a weight estimate as well as drug doses (based on the most appropriate weight-descriptor for drug type and habitus type) could reduce medication errors. This would improve the quality of care provided to obese children requiring emergency treatment.

\section{Putting it all together - science fiction or modern medicine?}

The described techniques might not be practical... yet! But derivative research may be able to establish their utility in both emergency and non-emergency settings and further refine their accuracy. The hightech interventions described in this study can easily be performed by emergency physicians, but the calculations involved are complex and the use of a smartphone app would be mandatory to ensure error-free translation of measurements to weight estimations and drug doses. Even the simplest systems are vulnerable to error if used incorrectly by untrained individuals, as has been shown with the Broselow tape [32-35] and clinicians would therefore need training dedicated to allow them to integrate weight-estimation into the management protocol. Even the use of PoCUS or BI could realistically be incorporated into team-based emergency care, with appropriate training.

The major factor in determining whether weight-estimation-drugdosing-technology remains science fiction or becomes a valid medical tool is the determination to minimise critical dosing errors in children.

\section{Limitations}

This was a very preliminary study with no separate validation sample. This data would therefore need to be verified in a future study and its potential role in weight estimation strategies critically evaluated. There was a relatively small number of obese children in which to develop and evaluate the FFM predictions, which will also require further research.

The practicality of these techniques in an emergency scenario was not evaluated.

\section{Conclusions}

This study confirmed that the use of length plus actual or surrogate anthropometric and high-tech measures of habitus allowed for accurate estimation of TBW, IBW and FFM. Point-of-care ultrasound and bioelectrical impedance measurements in combination with the PTXL significantly increased the accuracy of TBW and FFM estimations over methods using only anthropometric measurements. IBW was extremely accurately predicted by the PTXL and high-tech adjuncts could not increase the accuracy of the estimation. PoCUS, given how readily it is available in the Emergency Department, could have a role to play as an adjunct to the estimation of TBW and FFM in the future.

This study also showed that the PAWPER XL tape (using the habitus score or mid-arm circumference methodology) provided accurate estimations of TBW and IBW. With or without the use of high-tech adjuvants, this opens the door to more sophisticated and appropriate drug dosing for obese children and could reduce the perpetration of critical dosing errors in these children.

\section{Acknowledgements}

We would like to express our deep gratitude to Vertec South Africa who provided the Hologic Discovery A Bone Densitometer (Dualenergy X-ray Absorptiometry) machine on loan and to I Sandler \& Co. 
Wells M (2017) High-tech adjuncts to emergency weight estimation: Point-of-care ultrasound and point-of-care bioelectrical impedance measurements can increase the accuracy of length-based weight estimation in children

who provided the TANITA SC240MA body composition monitor on loan

\section{References}

1. Lubitz DS, Seidel JS, Chameides L, Luten RC, Zaritsky AL, et al. (1988) A rapid method for estimating weight and resuscitation drug dosages from length in the pediatric age group. Ann Emerg Med 17: 576-581. [Crossref]

2. Waters MR (1993) Paediatric resuscitation. Dosage inaccuracies may be dangerous. BMJ 306(6892): 1612-1613.

3. Srinivasan V, Nadkarni VM, Helfaer MA, Carey SM, Berg RA, et al. (2010) Childhood obesity and survival after in-hospital pediatric cardiopulmonary resuscitation. Pediatrics 125: e481-488. [Crossref]

4. Pinchevsky LE, Pesaturo KA, Smith BS, Hartman CA. (2010) Pilot comparison of three cardiopulmonary resuscitation medication dosing strategies in overweight children. $J$ Pediatr Pharmacol Ther. 15: 282-289. [Crossref]

5. Porter E, Barcega B, Kim TY. (2014) Analysis of medication errors in simulated pediatric resuscitation by residents. West J Emerg Med 15: 486-490. [Crossref]

6. Luscombe M (2005) "Kid's aren't what they used to be": a study of paediatric patients weights and their relationship to current weight estimation formulae. Brit J Anaest 95 : 578

7. Georgoulas V, Wells M (2016) The PAWPER tape and the Mercy Method outperform other methods of weight estimation in children in South Africa. S Afr Med J 106: 933 939. [Crossref]

8. Wells M, Coovadia A, Kramer E, Goldstein L (2013) The PAWPER tape: A new concept tape-based device that increases the accuracy of weight estimation in children through the inclusion of a modifier based on body habitus. Resuscitation 84: 227-232.

9. O’Leary F, John B, Hann L, Pegiazoglou I, McGarvey K (2016) Estimating weight in the Paediatric Emergency Department. Archives of Disease in Childhood. In press.

10. Sinha M, Lezine MW, Frechette A, Foster KN (2012) Weighing the pediatric patien during trauma resuscitation and its concordance with estimated weight using Broselow Luten emergency tape. Pediatric Emergency Care 28: 544-547.

11. Bierbrier R1, Lo V, Wu RC (2014) Evaluation of the accuracy of smartphone medical calculation apps. J Med Internet Res 16: e32. [Crossref]

12. Pineau JC, Lalys L, Pellegrini M, Battistini NC (2013) Body fat mass assessment: a comparison between an ultrasound-based device and a Discovery A model of DXA. International Scholarly Research Notices: Obesity. 2013: 462394.

13. Montagnese C, Williams JE, Haroun D, Siervo M, Fewtrell MS, et al. (2013) Is a single bioelectrical impedance equation valid for children of wide ranges of age, puberta status and nutritional status? Evidence from the 4-component model. Eur J Clin Nutr. 67 Suppl 1: S34-9.

14. Rowe S, Siegel D, Benjamin DK (2015) Best Pharmaceuticals for Children Act - Pediatric Trials Network Administrative Core C. Gaps in drug dosing for obese children: a systematic review of commonly prescribed emergency care medications. Clinical Therapeutics 37: 1924-1932.

15. Phillips S, Edlbeck A, Kirby M, Goday P (2007) Ideal body weight in children. Nutr Clin Pract 22: 240-245. [Crossref]

16. Abdel-Rahman SM, Ridge AL (2012) An improved pediatric weight estimation strategy. Open Medical Devices Journal 4: 87-97.
17. Chavez H, Peterson R, Lo K, Arel M (2015) Weight estimation in an inner city Pediatric Emergency Department: the effect of obesity. American Journal of Emergency Medicine 33: 1364-1367.

18. Ross EL, Heizer J, Mixon MA, Jorgensen J, Valdez CA, et al. (2015) Development of recommendations for dosing of commonly prescribed medications in critically ill obese children. Amer J Health-System Phar 72: 542-556.

19. Luten R, Zaritsky A (2008) The sophistication of simplicity... optimizing emergency dosing. Academic Emergency Medicine 15: 461-465.

20. Rush EC, Bristow S, Plank LD, Rowan J (2013) Bioimpedance prediction of fat-free mass from dual-energy X-ray absorptiometry in a multi-ethnic group of 2-year-old children. Eur J Clin Nutr 67: 214-217.

21. Traub SL, Kichen L (1983) Estimating ideal body mass in children. Am J Hosp Pharm 40: 107-110. [Crossref]

22. Traub SL, Johnson CE (1980) Comparison of methods of estimating creatinine clearance in children. Amer J Hosp Phar 37: 195-201.

23. Dieckmann RA (2007) The dilemma of paediatric drug dosing and equipment sizing in the era of patient safety. Emerg Med Australas 19: 490-493. [Crossref]

24. Foster BJ, Platt RW, Zemel BS (2012) Development and validation of a predictive equation for lean body mass in children and adolescents. Ann Hum Biol 39: 171-182. [Crossref]

25. Peters AM, Snelling HL, Glass DM, Bird NJ (2011) Estimation of lean body mass in children. Br J Anaesth 106: 719-723. [Crossref]

26. Pineau JC, Lalys L, Bocquet M, Guihard-Costa AM, Polak M, et al. (2010) Ultrasound measurement of total body fat in obese adolescents. Ann Nutr Metab 56: 36-44.

27. Kuperminc M (2009) Ultrasound imaging: a promising method for assessment of body composition. Dev Med \& Child Neur 51: 928.

28. Lammers R, Byrwa M, Fales W (2012) Root causes of errors in a simulated prehospital pediatric emergency. Acad Emer Med 19: 37-47.

29. Hoyle JD, Davis AT, Putman KK, Trytko JA, Fales WD (2012) Medication dosing errors in pediatric patients treated by emergency medical services. Prehosp Emer Care 16: 59-66.

30. Burden AR, Carr ZJ, Staman GW, Littman JJ, Torjman MC (2012) Does every code need a "reader?" improvement of rare event management with a cognitive aid "reader" during a simulated emergency: a pilot study. Simulation in Healthcare 7: 1-9.

31. Hawkes GA, Murphy G, Dempsey EM, Ryan AC (2015) Randomised controlled trial of a mobile phone infant resuscitation guide. J Paedi Child Health 51: 1084-8.

32. Lerner C, Gaca AM, Frush DP, Hohenhaus S, Ancarana A, et al. (2009) Enhancing pediatric safety: assessing and improving resident competency in life-threatening events with a computer-based interactive resuscitation tool. Pedia Radiol 39: 703-739.

33. Hohenhaus SM, Frush KS (2005) Pediatric patient safety in resuscitation simulations lessons learned from studying clinician behavior. Pediatric Emergency Care 21: 71-75.

34. McDaniel-Hohenhaus S, Frush K (2003) Development of an e-learning program for use of the Broselow-Luten pediatric system. J Emer Nur 29: 417.

35. Hohenhaus SM (2001) Is this a drill? Improving pediatric emergency preparedness in North Carolina's emergency departments. J Emer Nur 27: 568-70.

Copyright: (C2017 Wells M. This is an open-access article distributed under the terms of the Creative Commons Attribution License, which permits unrestricted use, distribution, and reproduction in any medium, provided the original author and source are credited. 